\title{
Levantamento florístico e sua distribuição diamétrica da vegetação de um cerrado sensu stricto e de um fragmento de floresta de galeria no ribeirão Dois Irmãos na APA de Cafuringa, DF, Brasil
}

\author{
José Elias de Paula \\ José Imaña-Encinas ${ }^{2 *}$ \\ Otacílio Antunes Santana ${ }^{3}$ \\ Gustavo Silva Ribeiro ${ }^{2}$ \\ Christian Rainier Imaña ${ }^{4}$ \\ ${ }^{1}$ Departamento de Botânica, Universidade de Brasília \\ ${ }^{2}$ Departamento de Engenharia Florestal, Universidade de Brasília \\ Caixa Postal 04457, CEP 70919-970, Brasília - DF, Brasil \\ ${ }^{3}$ Departamento de Geografia, Universidade de Brasília \\ ${ }^{4}$ Secretaria da Fazenda do Estado de Minas Gerais \\ *Autor para correspondência \\ imana@unb.br
}

Submetido em 30/11/2008

Aceito para publicação em 13/03/2009

\section{Resumo}

Foram identificadas todas as espécies vegetais ocorrentes em uma área de $2.500 \mathrm{~m}^{2}$ de cerrado sensu stricto e de $5.000 \mathrm{~m}^{2}$ de um fragmento de uma floresta de galeria do ribeirão Dois Irmãos na APA de Cafuringa, localizada na região norte do Distrito Federal, próximo à divisa do Estado de Goiás, nas coordenadas geográficas 15³0'19”S e 48 $06^{\prime} 18^{\prime \prime}$. Foram medidos os diâmetros de todos os indivíduos arbóreos com diâmetro à altura do peito (DAP) a partir de $5 \mathrm{~cm}$, tanto do cerrado sensu stricto como do fragmento da floresta de galeria. A composição florística do cerrado sensu stricto ficou composta por 100 indivíduos arbóreos distribuídos em 25 espécies, e da floresta de galeria por 155 árvores distribuídas em 50 espécies. A regeneração natural constituída por indivíduos de hábito arbóreo com DAP inferior a $5 \mathrm{~cm}$, no cerrado sensu stricto foi de 211 indivíduos distribuídos em 38 espécies, e na floresta de galeria foram 287 indivíduos, distribuídos em 55 espécies. A área basal ocupada pelos componentes arbóreos foi de $3,40 \mathrm{~m}^{2}$.ha-1 na área do cerrado sensu stricto e $5,08 \mathrm{~m}^{2} . \mathrm{ha}^{-1}$ na floresta de galeria. A curva da distribuição diamétrica de ambas as comunidades ajustada pela equação de Meyer apresentou a tendência típica do "J" invertido e mostrou forte intervenção antrópica nas classes de $11 \mathrm{a} 17 \mathrm{~cm}$.

Unitermos: análise dendrométrica, composição florística, inventário florestal, regeneração natural

\section{Abstract}

Survey of vegetation and its diametric distribution in an area of cerrado sensu stricto and riparian forest fragment at Dois Irmãos stream in the Area of Environmental Protection (APA) of Cafuringa, 
Federal District, Brazil. All individual trees with a diameter at breast height (DBH) of over $5 \mathrm{~cm}$, as well as the natural succession, were identified in $2,500 \mathrm{~m}^{2}$ of the savannah (cerrado sensu stricto) area and in $5,000 \mathrm{~m}^{2}$ of the “Dois Irmãos" riparian forest vegetation (15³0'19"S and 48 $06^{\prime} 18^{\prime}$ ”W). The floristic composition of the cerrado sensu stricto was composed by 100 trees distributed in 25 species, and the riparian forest consisted of 155 trees distributed in 55 species. The natural regeneration was formed with 211 and 287 individuals in the cerrado sensu stricto and riparian forest distributed into 38 and 55 species respectively. The basal areas of the trees occupied $3.40 \mathrm{~m}^{2}$.ha $\mathrm{a}^{-1}$ in the cerrado sensu stricto and $5.08 \mathrm{~m}^{2} . \mathrm{ha}^{-1}$ in the riparian forest. The diametric distribution curves for both plant communities, adjusted by the Meyers equation, demonstrated a typical tendency of reversed-J shape with strongly antropic action in the 11 to $17 \mathrm{~cm}$ diametric classes.

Key words: dendrometrical analysis, floristic composition, forest inventory, natural regeneration

\section{Introdução}

O Bioma Cerrado, segundo maior do Brasil, cobre uma superfície próxima a dois milhões de $\mathrm{km}^{2}$, dos quais cerca de $60 \%$ já foram destruídos (UNESCO, 2000; Santos e Câmara, 2002). A UNESCO, em 1993 estabeleceu a Reserva da Biosfera do Cerrado, como forma de preservar áreas desse bioma. Parte dessa reserva está localizada no Distrito Federal, cobrindo uma área de 226 mil hectares, que correspondem a 30\% da área do Distrito Federal. A Área de Proteção Ambiental de Cafuringa, na divisa do Distrito Federal com o estado de Goiás, é uma das unidades de preservação ambiental da Reserva da Biosfera do Cerrado (SEMARH, 2005). Naquele local, na região da sub-bacia hidrográfica do ribeirão Dois Irmãos, encontra-se uma região escarpada com encostas acentuadas cobertas por uma vegetação natural, regionalmente conhecida como área da Dolina da Garapa (SEMARH, 2005). Nela se encontra a caverna da Garapa, na fazenda Portal dos Angicos. Deu-se esse nome à caverna pela presença de um indivíduo de grande porte de Garapa (Apuleia leiocarpa (Vogel) J.F. Macbr.). A vegetação natural nessa região está composta por fragmentos de florestas de galeria circundadas naturalmente pelo cerrado sensu stricto.

No intuito de dar subsídios aos planos de manejo e de preservação da biodiversidade da APA de Cafuringa (Distrito Federal), realizou-se um levantamento da composição florística da vegetação natural do cerrado sensu stricto e de um fragmento de floresta de galeria de uma vertente do ribeirão Dois Irmãos, localizadas na APA mencionada, assim como identificar a correspondente distribuição diamétrica dos indivíduos arbóreos.

\section{Material e Métodos}

Segundo os estudos realizados pela Secretaria do Meio Ambiente e Recursos Hídricos do Governo do Distrito Federal (SEMARH, 2005) a área da sub-bacia hidrográfica do ribeirão Dois Irmãos cobre uma superfície de $32 \mathrm{~km}^{2}$, caracterizada pelo tipo de clima Aw da classificação de Köppen, apresentando uma temperatura média anual de $21,2^{\circ} \mathrm{C}$ com uma precipitação em torno de $1.552 \mathrm{~mm} \cdot$ ano $^{-1}$. A sub-bacia hidrográfica do ribeirão Dois Irmãos fica localizada na região norte da APA de Cafuringa, no Distrito Federal.

Entre duas encostas de elevada declividade (entre 45 e $65^{\circ}$ ) se localiza uma vertente do ribeirão Dois Irmãos e uma grota estreita que drena suas águas para o interior de uma caverna conhecida como caverna da Garapa. No fundo desse vale, formado por uma depressão de mais de $100 \mathrm{~m}$ de comprimento com uma profundidade de mais de $25 \mathrm{~m}$, as copas das árvores das duas margens da vertente ao ribeirão Dois Irmãos se sobrepõem formando uma floresta de galeria. Circundando a floresta de galeria, nas duas encostas se localiza o cerrado sensu stricto.

No cerrado sensu stricto, a área de estudo foi uma parcela retangular de $250 \times 10 \mathrm{~m}\left(2.500 \mathrm{~m}^{2}\right)$ nas coordenadas geográficas $15^{\circ} 30^{\prime} 19^{\prime \prime} \mathrm{S}$ e $48^{\circ} 06^{\prime} 18^{\prime \prime} \mathrm{W}$, e altitude entre 909 e $921 \mathrm{~m}$. A parcela da floresta de galeria foi uma faixa de $10 \times 500 \mathrm{~m}\left(5.000 \mathrm{~m}^{2}\right)$. Nessas parcelas, foram identificadas todas as espécies arbóreas, arbustivas, ervas e trepadeiras. Árvore foi considerado o indivíduo com diâmetro à altura do peito (DAP) igual ou superior a $5 \mathrm{~cm}$. As parcelas foram divididas em subparcelas de 1 x 10m e nelas foi realizado o levantamento da regeneração natural. Nesse levantamento ingressaram os indivíduos com DAP inferior a $5 \mathrm{~cm}$. Arbusto foi 
o vegetal lenhoso que não formava fuste e nem copa definida, seja qual for a sua altura. Erva foi o indivíduo de consistência herbácea cujo hábito de crescimento era ereto sem a formação de lenho, e trepadeira, indivíduo de consistência lenhosa sem sustentação própria crescendo junto aos fustes das árvores. As espécies de gramíneas (Poaceae) não foram consideradas. A forma de vida da espécie ficou atrelada à classificação da vegetação apresentada na obra APA de Cafuringa: a última fronteira natural do DF (SEMARH, 2005).

A grafia dos nomes científicos das espécies e a identificação de família foi verificada pela nomenclatura Trópicos disponível no site do Jardim Botânico de Missouri (TROPICOS, 2008). O material fértil coletado que serviu de base para a identificação dos binômios, foi depositado, em correspondentes exsicatas, no Herbário UB, da Universidade de Brasília, sob correspondente número de coleta.

Em todos os indivíduos arbóreos foi medido o DAP, tanto na parcela do cerrado sensu stricto como da floresta de galeria. Como instrumento foi utilizado uma suta dendrométrica de $80 \mathrm{~cm}$ de comprimento. $\mathrm{A}$ área basal $(\mathrm{AB})$ foi determinada pela fórmula: $\mathrm{AB}=\mathrm{DAP}^{2} \cdot 0,7854$.

Para o estudo da estrutura diamétrica das árvores, o DAP medido, das espécies do cerrado sensu stricto e da floresta de galeria, foi distribuído em classes de diâmetro com intervalos de $2 \mathrm{~cm}$, ficando a curva correspondente ajustada ao modelo conhecido como exponencial negativo de Meyer (1952) $y=\mathrm{b}_{0} \cdot e^{\mathrm{b}_{1} x}$. Os valores do coeficiente de determinação $\left(\mathrm{R}^{2}\right)$, nível de significância $(P)$ e o erro do ajuste da equação considerada foram calculados com auxílio do programa Statistica 5.1 (StatSoft, 1997).

\section{Resultados e Discussão}

No cerrado sensu stricto foram encontrados 100 indivíduos arbóreos, pertencentes a 25 espécies. Além das espécies arbóreas foram registradas treze espécies arbustivas, três espécies trepadeiras e quinze espécies herbáceas (Tabela 1).
Na regeneração natural foram amostrados 211 indivíduos arbóreos pertencentes a 38 espécies arbóreas (Tabela 2). Comparativamente, na região do Distrito Federal, foram encontrados em áreas de cerrado sensu stricto, entre 49 (Rezende et al., 2006) e 72 espécies arbóreas (Felfili et al., 1993), valores superiores aos do presente estudo.

$\mathrm{Na}$ floresta de galeria foram amostrados 155 indivíduos, distribuídos em 50 espécies (Tabela 1). Além dessas espécies arbóreas foram encontradas três espécies arbustivas, uma espécie trepadeira e quatro espécies herbáceas. Na análise da regeneração natural da floresta de galeria foram constatados 287 indivíduos arbóreos sucessores, com DAP inferior a $5 \mathrm{~cm}$ (Tabela 2) distribuídos em 55 espécies arbóreas. Felfili (1997) registrou para a floresta de galeria do Gama (DF) 93 espécies arbóreas, e Silva Júnior (2005a) na mata de galeria do Pitoco (DF) listou 99 espécies. Paula et al. (1990) identificaram 82 espécies para a floresta de galeria do rio São Bartolomeu (DF). Em situação homóloga ao cerrado sensu stricto avaliado neste estudo, o número de espécies encontradas para a floresta de galeria foi menor do que áreas da mesma tipologia na região, provavelmente por ter empregado um esforço amostral menor aos trabalhos comparados.

Os 100 indivíduos arbóreos do cerrado sensu stricto foram analisados em função do DAP médio aritmético e área basal, apresentando respectivamente os valores de $8,96 \mathrm{~cm}$ e $3,40 \mathrm{~m}^{2}$. ha $^{-1}$ (Tabela 1). Felfili et al. (2004) aferiram em três áreas de cerrado sensu stricto na região do Distrito Federal valores entre 5,79 e $10,76 \mathrm{~m}^{2} \cdot \mathrm{ha}^{-1}$. Para um fragmento de cerrado sensu stricto na APA do Paranoá (DF) foi descrita uma área basal de $9,53 \mathrm{~m}^{2} \cdot$ ha $^{-1}$ (Assunção e Felfili, 2004). Rezende et al. (2006) registraram $6,23 \mathrm{~m}^{2} . \mathrm{ha}^{-1}$ para uma área semelhante na Fazenda da Universidade de Brasília (DF). Paula et al. (1998) e Imaña-Encinas e Paula (2003), estudando três hectares de cerrado sensu stricto no Estado do Maranhão, registraram áreas basais entre 6,15 e $6,67 \mathrm{~m}^{2} \mathrm{ha}^{-1}$. Comparando as áreas basais encontradas na literatura, se evidencia que a do cerrado sensu stricto analisado é muito baixa. 
O DAP médio de duas espécies da comunidade cerrado sensu stricto foi superior a $20 \mathrm{~cm}$. Ficou evidente que se trata de uma comunidade vegetal de pequeno porte, uma vez que $88 \%$ dos indivíduos arbóreos apresentaram DAP médio inferior a $11 \mathrm{~cm}$ (Tabela 1). Os 100 espécimes arbóreos da comunidade cerrado sensu stricto ordenados em uma distribuição diamétrica de $2 \mathrm{~cm}$, apresentaram curva de decaimento típica para comunidades vegetais (Figura 1). A distribuição diamétrica mostrou-se irregular, nas primeiras classes, permitindo inferir que a comunidade vegetal já teve provavelmente forte intervenção antrópica ou natural, refletida principalmente nas classes diamétricas de 11 a $17 \mathrm{~cm}$, mostrando a existência de menos indivíduos do esperado pela curva de ajuste.

TABELA 1: Composição florística, forma de vida e parâmetros dendrométricos do cerrado sensu stricto e do fragmento da floresta de galeria do ribeirão Dois Irmãos, na APA de Cafuringa $-\mathrm{DF}$. ( $\mathrm{N}=$ número de indivíduos amostrados, DAP = diâmetro à altura do peito)

\begin{tabular}{|c|c|c|c|c|c|c|c|}
\hline \multirow{2}{*}{ Familia/ Espécie } & \multirow[b]{2}{*}{$\begin{array}{c}\text { Forma } \\
\text { de vida }\end{array}$} & \multicolumn{3}{|c|}{ Cerrado sensu stricto ${ }^{1}$} & \multicolumn{3}{|c|}{ Floresta de galeria $^{2}$} \\
\hline & & $\mathrm{N}$ & $\begin{array}{l}\text { DAP } \\
\text { médio } \\
(\mathrm{cm})\end{array}$ & $\begin{array}{c}\text { Área } \\
\text { basal } \\
\left(\mathrm{m}^{2} \mathrm{ha}^{-1}\right)\end{array}$ & $\mathrm{N}$ & $\begin{array}{l}\text { DAP } \\
\text { médio } \\
(\mathrm{cm})\end{array}$ & $\begin{array}{c}\text { Área } \\
\text { basal } \\
\left(\mathrm{m}^{2} \mathrm{ha}^{-1}\right)\end{array}$ \\
\hline \multicolumn{8}{|l|}{ Anacardiaceae } \\
\hline Anacardium humile A. St.-Hil. & arbusto $^{1}$ & & & & & & \\
\hline Astronium fraxinifolium Schott & árvore & 7 & 8,31 & 0,18102 & & & \\
\hline Tapirira guianensis Aublet & árvore & & & & 3 & 23,2 & 0,3006 \\
\hline \multicolumn{8}{|l|}{ Annonaceae } \\
\hline Annona monticola Mart. & arbusto $^{1}$ & & & & & & \\
\hline Xylopia brasiliensis Spreng. & árvore & & & & 8 & 9,8 & 0,1495 \\
\hline \multicolumn{8}{|l|}{ Apocynaceae } \\
\hline Aspidosperma australe Müll. Arg. & árvore & & & & 2 & 11,2 & 0,0413 \\
\hline Aspidosperma subincanum Mart. ex A. DC. & árvore & & & & 2 & 18 & 0,1272 \\
\hline Himatanthus obovatus (Müll. Arg.) Woodson & árvore & 3 & 8,5 & 0,0739 & & & \\
\hline Mandevilla hirsuta (Rich.) K. Schum. & trepadeira ${ }^{1}$ & & & & & & \\
\hline \multicolumn{8}{|l|}{ Araceae } \\
\hline Philodendron lundii Warm. & erva $^{1}$ & & & & & & \\
\hline \multicolumn{8}{|l|}{ Arecaceae } \\
\hline Geonoma pohliana Mart. & árvore & & & & 2 & 7,2 & 0,0181 \\
\hline Syagrus romanzoffiana (Cham.) Glassman & árvore & & & & 1 & 8,5 & 0,0113 \\
\hline \multicolumn{8}{|l|}{ Asteraceae } \\
\hline Aspilia floribunda (Gardner) Baker & erva $^{1}$ & & & & & & \\
\hline Eremanthus glomerulatus Less. & arbusto $^{1}$ & & & & & & \\
\hline Ichthyothere latifólia & erva $^{1}$ & & & & & & \\
\hline Riencourtia oblongifolia Gardner & erva $^{1}$ & & & & & & \\
\hline \multicolumn{8}{|l|}{ Bignoniaceae } \\
\hline Arrabidaea brachypoda (DC.) Bureau & arbusto $^{1}$ & & & & & & \\
\hline Handroanthus impetiginosus (Mart. ex DC.) Mattos & árvore & & & & 2 & 7,2 & 0,0165 \\
\hline Zeyheria digitalis (Vell.) L.B. Sm. \& Sandwith & arbusto $^{1}$ & & & & & & \\
\hline \multicolumn{8}{|l|}{ Boraginaceae } \\
\hline Cordia rufescens A. DC. & árvore & & & & 1 & 19 & 0,0567 \\
\hline \multicolumn{8}{|l|}{ Bromeliaceae } \\
\hline Ananas ananassoides (Baker) L.B. Sm. & erva $^{1}$ & & & & & & \\
\hline Dyckia brachyphylla L.B. Sm. & erva $^{1}$ & & & & & & \\
\hline
\end{tabular}




\begin{tabular}{|c|c|c|c|c|c|c|c|}
\hline \multicolumn{8}{|l|}{ Burseraceae } \\
\hline Protium heptaphyllum (Aubl.) Marchand & árvore & & & & 11 & 14,9 & 0,4463 \\
\hline Protium spruceanum (Benth.) Engl. & árvore & 1 & 7 & 0,0154 & & & \\
\hline \multicolumn{8}{|l|}{ Chrysobalanaceae } \\
\hline Hirtella glandulosa Spreng. & árvore & & & & 2 & 6,7 & 0,0145 \\
\hline Licania apetala (E. Mey.) Fritsch & árvore & & & & 5 & 8,7 & 0,0694 \\
\hline \multicolumn{8}{|l|}{ Clusiaceae } \\
\hline Kielmeyera coriacea Mart. \& Zucc. & árvore & & & & 1 & 8 & 0,0100 \\
\hline \multicolumn{8}{|l|}{ Connaraceae } \\
\hline Connarus suberosus Planch. & árvore & 4 & 9,1 & 0,1108 & 2 & 7 & 0,0157 \\
\hline \multicolumn{8}{|l|}{ Cucurbitaceae } \\
\hline Gurania spinulosa (Poepp. \& Endl.) Cogn. & erva $^{2}$ & & & & & & \\
\hline \multicolumn{8}{|l|}{ Cyperaceae } \\
\hline Scleria scabra Willd. & erva $^{1}$ & & & & & & \\
\hline Rhynchospora exaltata Kunth & erva $^{2}$ & & & & & & \\
\hline \multicolumn{8}{|l|}{ Dilleniaceae } \\
\hline Curatella americana $\mathrm{L}$. & árvore & 1 & 10,5 & 0,0346 & & & \\
\hline \multicolumn{8}{|l|}{ Erythroxylaceae } \\
\hline Erythroxylum daphnites Mart. & árvore & 9 & 7,39 & 0,1669 & 8 & 7,8 & 0,0852 \\
\hline Erythroxylum suberosum A. St.-Hil. & arbusto ${ }^{1}$ & & & & & & \\
\hline Erythroxylum vacciniifolium Mart. & árvore & & & & 2 & 5,5 & 0,0095 \\
\hline \multicolumn{8}{|l|}{ Euphorbiaceae } \\
\hline Croton antisyphiliticus Mart. & erva $^{1}$ & & & & & & \\
\hline Manihot longipetiolata Pohl & arbusto $^{1}$ & & & & & & \\
\hline \multicolumn{8}{|l|}{ Fabaceae } \\
\hline Acosmium glaziovianum (Harms) Yakovlev & árvore & 2 & 7,65 & 0,0368 & & & \\
\hline Anadenanthera colubrina (Vell.) Brenan & árvore & & & & 5 & 31,7 & 0,3156 \\
\hline Apuleia leiocarpa (Vogel) J.F. Macbr. & árvore & & & & 4 & 23,5 & 0,1754 \\
\hline Bauhinia depauperata Glaz. & arbusto ${ }^{1}$ & & & & & & \\
\hline Bauhinia rufa (Bong.) Steud. & árvore & 1 & 5,3 & 0,0088 & & & \\
\hline Bowdichia virgilioides Kunth & árvore & 8 & 10,18 & 0,4420 & 3 & 21,5 & 0,2193 \\
\hline Copaifera langsdorffii Desf. & árvore & & & & 2 & 13,7 & 0,0694 \\
\hline Eriosema prorepens Benth. & erva $^{1}$ & & & & & & \\
\hline Hymenaea courbaril L. & árvore & & & & 3 & 22,8 & 0,2781 \\
\hline Hymenaea stigonocarpa Mart. ex Hayne & árvore & 4 & 14,5 & 0,2937 & & & \\
\hline Inga nobilis Willd. & árvore & & & & 2 & 17 & 0,1109 \\
\hline Machaerium opacum Vogel & árvore & 1 & 33 & 0,3421 & & & \\
\hline Stylosanthes guianensis (Aubl.) Sw. & erva $^{1}$ & & & & & & \\
\hline Vatairea macrocarpa (Benth.) Ducke & árvore & 1 & 9,5 & 0,0283 & & & \\
\hline \multicolumn{8}{|l|}{ Gentianaceae } \\
\hline Curtia tenuifolia (Aubl.) Knobl. & erva $^{1}$ & & & & & & \\
\hline \multicolumn{8}{|l|}{ Icacinaceae } \\
\hline Emmotum nitens (Benth.) Miers & árvore & & & & 2 & 9 & 0,0261 \\
\hline \multicolumn{8}{|l|}{ Iridaceae } \\
\hline Cipura paludosa Aubl. & erva $^{1}$ & & & & & & \\
\hline
\end{tabular}




\begin{tabular}{|c|c|c|c|c|c|c|c|}
\hline \multicolumn{8}{|l|}{ Lamiaceae } \\
\hline Hyptidendron canum (Pohl ex Benth.) Harley & árvore & 28 & 9,31 & 0,8865 & & & \\
\hline \multicolumn{8}{|l|}{ Lauraceae } \\
\hline Ocotea corymbosa (Meisn.) Mez & árvore & & & & 17 & 11,2 & 0,4290 \\
\hline Ocotea pomaderroides (Meisn.) Mez & árvore & & & & 6 & 16,9 & 0,2809 \\
\hline \multicolumn{8}{|l|}{ Malpighiaceae } \\
\hline Banisteriopsis anisandra (A. Juss.) B. Gates & trepadeira $^{1}$ & & & & & & \\
\hline Byrsonima coccolobifolia Kunth & árvore & 2 & 5,75 & 0,0208 & & & \\
\hline Byrsonima crassa Nied. & árvore & 2 & 6,6 & 0,0274 & & & \\
\hline Byrsonima intermedia A. Juss. & árvore & & & & 1 & 7 & 0,0077 \\
\hline \multicolumn{8}{|l|}{ Malvaceae } \\
\hline Luehea divaricata Mart. & árvore & & & & 1 & 5,5 & 0,0047 \\
\hline Pseudobombax longiflorum (Mart. \& Zucc.) A. Robyns & árvore & 1 & 21 & 0,1385 & 5 & 29,3 & 0,7914 \\
\hline \multicolumn{8}{|l|}{ Melastomataceae } \\
\hline Miconia fallax DC. & arbusto $^{1}$ & & & & & & \\
\hline \multicolumn{8}{|l|}{ Monimiaceae } \\
\hline Siparuna guianensis Aubl. & árvore & & & & 2 & 7,5 & 0,0184 \\
\hline \multicolumn{8}{|l|}{ Moraceae } \\
\hline Ficus obtusifolia (Miq.) Miq. & árvore & & & & 2 & 21 & 0,1414 \\
\hline \multicolumn{8}{|l|}{ Myrtaceae } \\
\hline Eugenia aurata O. Berg. & árvore & 2 & 5,75 & 0,0208 & 2 & 18 & 0,1131 \\
\hline Eugenia bracteata Rich. & arbusto $^{1}$ & & & & & & \\
\hline Eugenia dysenterica DC. & árvore & 1 & 8,5 & 0,0227 & & & \\
\hline Gomidesia lindeniana $\mathrm{O}$. Berg. & árvore & & & & 3 & 7,5 & 0,0281 \\
\hline Myrcia fallax (Rich.) DC. & árvore & 4 & 7,55 & 0,0735 & 3 & 6,3 & 0,0191 \\
\hline Myrcia lindeniana $\mathrm{O}$. Berg. & árvore & & & & 3 & 7,3 & 0,0261 \\
\hline Myrcia tomentosa (Aubl.) DC. & árvore & 7 & 9,34 & 0,2230 & 2 & 8 & 0,0204 \\
\hline Psidium australe Cambess. & arbusto $^{1}$ & & & & & & \\
\hline \multicolumn{8}{|l|}{ Nyctaginaceae } \\
\hline Neea theifera Oerst. & árvore & 1 & 10,5 & 0,0346 & 3 & 14,2 & 0,0682 \\
\hline \multicolumn{8}{|l|}{ Ochnaceae } \\
\hline Ouratea spectabilis (Mart. \& Engl.) Engl. & árvore & & & & 1 & 6,5 & 0,0066 \\
\hline \multicolumn{8}{|l|}{ Oxalidaceae } \\
\hline Oxalis hirsutissima Mart. ex Zucc. & erva $^{1}$ & & & & & & \\
\hline \multicolumn{8}{|l|}{ Piperaceae } \\
\hline Piper arboreum Aubl. & arbusto $^{2}$ & & & & & & \\
\hline \multicolumn{8}{|l|}{ Polygalaceae } \\
\hline Polygala cuspidata DC. & erva $^{1}$ & & & & & & \\
\hline \multicolumn{8}{|l|}{ Proteaceae } \\
\hline Euplassa inaequalis (Pohl) Engl. & árvore & & & & 1 & 8 & 0,0100 \\
\hline \multicolumn{8}{|l|}{ Rubiaceae } \\
\hline Amaioua guianensis Aubl. & árvore & & & & 3 & 10,7 & 0,0701 \\
\hline Cordiera macrophylla Kuntze. & árvore & & & & 2 & 7,7 & 0,0189 \\
\hline Guettarda viburnoides Cham. \& Schltdl. & árvore & & & & 3 & 8,2 & 0,0318 \\
\hline Palicourea rigida Kunth & arbusto $^{1}$ & & & & & & \\
\hline Palicourea squarrosa (Müll. Arg.) Standl. & arbusto $^{2}$ & & & & & & \\
\hline
\end{tabular}


Psychotria capitata Ruiz \& Pav.

Psychotria sciaphila S. Moore

Sabicea brasiliensis Wernham

Tocoyena formosa (Cham. \& Schltdl.) K. Schum.

Tocoyena velutina Mart.

Salicaceae

Casearia grandiflora Cambess.

Casearia sylvestris $\mathrm{Sw}$. var. sylvestris

Sapindaceae

Cupania vernalis Cambess.

Matayba guianensis Aubl.

Serjania lethalis A. St.-Hil.

\section{Sapotaceae}

Micropholis venulosa (Mart. \& Eichler) Pierre

Smilacaceae

Smilax fluminensis Steud.

Styracaceae

\begin{tabular}{|c|c|c|c|c|c|c|c|}
\hline Styrax camporum Pohl & árvore & & & & 7 & 8,8 & 0,0921 \\
\hline \multicolumn{8}{|l|}{ Jrticaceae } \\
\hline Cecropia pachystachya Trécul & árvore & & & & 1 & 15 & 0,0353 \\
\hline \multicolumn{8}{|l|}{ Terbenaceae } \\
\hline Aegiphila paraguariensis Briq. & árvore & & & & 1 & 9 & 0,0127 \\
\hline \multicolumn{8}{|l|}{ ochysiaceae } \\
\hline Callisthene major Mart. & árvore & & & & 1 & 31 & 0,1509 \\
\hline Qualea grandiflora Mart. & árvore & 1 & 6,6 & 0,0137 & 2 & 13,7 & 0,0808 \\
\hline Qualea parviflora Mart. & árvore & 3 & 10,07 & 0,1072 & & & \\
\hline otal & & 100 & & 3,4013 & 155 & & 5,0866 \\
\hline
\end{tabular}

${ }^{1}$ espécies encontradas na fitofisionomia Cerrado sensu stricto, ${ }^{2}$ na floresta de galeria.

TABELA 2: Número de indivíduos por hectare das espécies da regeneração natural do cerrado sensu stricto e floresta de galeria do ribeirão Dois Irmãos na APA de Cafuringa - Distrito Federal.

\begin{tabular}{|c|c|c|c|}
\hline Familia / Espécie & $\begin{array}{c}\text { Cerrado } \\
\text { sensu stricto } \\
\text { ha }\end{array}$ & $\begin{array}{c}\text { Floresta de } \\
\text { galeria } \\
\text { ha }\end{array}$ & Característica de uso \\
\hline \multicolumn{4}{|l|}{ Anacardiaceae } \\
\hline Astronium fraxinifolium Schott & 16 & 6 & Planta medicinal e alimento para a fauna (3) \\
\hline Tapirira guianensis Aublet & & 26 & Alimento para a fauna (3) \\
\hline \multicolumn{4}{|l|}{ Annonaceae } \\
\hline Xylopia brasiliensis Spreng. & & 52 & Alimento para a fauna e paisagismo $(1,5)$ \\
\hline \multicolumn{4}{|l|}{ Apocynaceae } \\
\hline Aspidosperma australe Müll. Arg. & & 8 & Planta medicinal e arborização (2) \\
\hline Himatanthus obovatus (Müll. Arg.) Woodson & 8 & 8 & Planta medicinal. (2) paisagismo (5) \\
\hline \multicolumn{4}{|l|}{ Araliaceae } \\
\hline Didymopanax macrocarpon (Cham. \& Schltdl.) Seem. & 8 & & Alimento para a fauna (2) \\
\hline \multicolumn{4}{|l|}{ Arecaceae } \\
\hline Geonoma pohliana Mart. & & 8 & Planta ornamental \\
\hline
\end{tabular}


Syagrus romanzoffiana (Cham.) Glassman

\section{Bignoniaceae}

Tabebuia impetiginosa (Mart. ex DC.) Standl.

Tabebuia ochracea (Cham.) Standl.

Bombacaceae

Pseudobombax longiflorum (Mart. \& Zucc.) A. Robyns

Boraginaceae

Cordia rufescens A. DC.

Burseraceae

Protium spruceanum (Benth.) Engl.

Chrysobalanaceae

Hirtella glandulosa Spreng.

Licania apetala (E. Mey.) Fritsch

Clusiaceae

Kielmeyera coriacea Mart. \& Zucc.

Vismia decipiens Schltdl. \& Cham.

Combretaceae

Terminalia glabrescens Mart.

Connaraceae

Connarus suberosus Planch.

Dichapetalaceae

Tapura amazonica Poepp.

Dilleniaceae

Curatella americana $\mathrm{L}$.

Erythroxylaceae

Erythroxylum daphnites Mart.

Erythroxylum vacciniifolium Mart.

Fabaceae

Acosmium glaziovianum (Harms) Yakovlev

Anadenanthera colubrina (Benth.) Brenan

Apuleia leiocarpa (Vogel) J.F. Macbr.

Bauhinia rufa (Bong.) Steud.

Bowdichia virgilioides Kunth

Copaifera langsdorffii Desf.

Hymenaea courbaril L.

Hymenaea stigonocarpa Mart. ex Hayne

Inga nobilis Willd.

Machaerium opacum Vogel

Piptadenia communis Benth.

Vatairea macrocarpa (Benth.) Ducke

Icacinaceae

Emmotum nitens (Benth.) Miers

Lamiaceae

Hyptidendron canum (Pohl) Harley

Lauraceae

Ocotea corymbosa (Meisn.) Mez

Ocotea pomaderroides (Meisn.) Mez

Loganiaceae

Antonia ovata Pohl

Malpighiaceae

Byrsonima coccolobifolia Kunth

Byrsonima crassa Nied.
4

Alimento para a fauna e paisagismo (1)

$4 \quad$ Planta medicinal e paisagismo $(3,5)$

2 Paisagismo (5)

6 Produção de fibras (4)

$4 \quad$ Planta medicinal (6)

$32 \quad 56 \quad$ Alimento para a fauna (2), paisagismo (5)

24 Alimento para a fauna (2)

20 Planta medicinal (5)

8

4

Planta medicinal e melífera (4)

Planta medicinal (5)

4 Produção de madeira e arborização (3)

8 $\quad$ Planta medicinal e alimento para a fauna (2)

$16 \quad$ Alimento para a fauna (3)

4

Planta medicinal (3), alimento para a fauna (1)

$48 \quad 10 \quad$ Alimento para a fauna $(3,5)$

8 Alimento para a fauna $(3,5)$

56 Produção de madeira $(3,5)$

10 Produção de madeira e paisagismo (1)

6 Produção de madeira e paisagismo (1)

12

1614 Produção de madeira e paisagismo (1)

6 Produção de madeira, óleo e planta medicinal (1)

14 Produção de madeira e alimento para a fauna (1)

12 Madeira $\mathrm{p} /$ embarcações, alimento para a fauna (1)

$4 \quad$ Alimento para a fauna (5)

4 Alimento para a fauna, produção de carvão (2)

14 Melífera e madeira para carvão e lenha (1)

14 Madeira para construção e planta medicinal (2)

124 Produção de madeira e alimento para a fauna (2)

$44 \quad$ Planta medicinal (3)

12 Produção madeira para construção e arborização (1)

10 Planta medicinal

Produção de madeira e planta medicinal (3)

12

Alimento para a fauna (2)

Produção de madeira 
Byrsonima intermedia A. Juss.

Malvaceae

Luehea divaricata Mart.

Melastomataceae

Miconia fallax DC.

Meliaceae

Cabralea canjerana (Vell.) Mart.

Monimiaceae

Siparuna guianensis Aubl.

Myristicaceae

Virola sebifera Aubl.

Myrtaceae

Eugenia aurata $\mathrm{O}$. Berg.

Eugenia dysenterica DC.

Gomidesia lindeniana O. Berg.

Myrcia fallax (Rich.) DC.

Myrcia tomentosa (Aubl.) DC.

Nyctaginaceae

Neea theifera Oerst.

Ochnaceae

Ouratea spectabilis (Mart. \& Engl.) Engl.

Proteaceae

Euplassa inaequalis (Pohl) Engl.

Rubiaceae

Alibertia edulis (Rich.) A. Rich. ex DC.

Amaioua guianensis Aubl.

Cordiera macrophylla Kuntze.

Guettarda viburnoides Cham. \& Schltdl.

Tocoyena formosa (Cham. \& Schltdl.) K. Schum.

Tocoyena velutina Mart.

Salicaceae

Casearia grandiflora Cambess.

Casearia sylvestris Sw.

68

Sapindaceae

Cupania vernalis Cambess.

Matayba guianensis Aubl.

Sapotaceae

Micropholis venulosa (Mart. \& Eichler) Pierre

Pouteria gardneri (Mart. \& Miq.) Baehni

Simaroubaceae

Simarouba versicolor A. St.-Hil.

Styracaceae

Styrax camporum Pohl

Ulmaceae

Celtis brasiliensis (Gardner) Planch.

Verbenaceae

Aegiphila paraguariensis Briq.

Vochysiaceae

Qualea grandiflora Mart.

Qualea parviflora Mart.

Salvertia convallariodora A. St.-Hil.

Vochysia elliptica Mart.

Total de indivíduos

60

24

28

2
$12 \quad$ Planta medicinal (7)

8 Produção de madeira. Planta medicinal (3)

Produção de madeira (lenha)

8 Alimento para a fauna. (1), madeira nobre

$18 \quad$ Planta medicinal

$4 \quad$ Alimento para a fauna. (1) planta medicinal (3)

$40 \quad$ Alimento para a fauna

Planta medicinal e alimento para a fauna (2)

1212 Produção de madeira e alimento para a fauna (2)

12

14 Produção de madeira e alimento para a fauna (2)

$16 \quad 6 \quad$ Planta medicinal e alimento para a fauna (4)

2 Produção de madeira e alimento para a fauna (2)

6 Produção de madeira e alimento para a fauna (3)

$68 \quad$ Paisagismo (6)

6 Produção de madeira e alimento para a fauna (2)

8 Alimento para a fauna

86 Produção de madeira e alimento para a fauna (2)

36 Paisagismo e forrageira (4)

6

14 Planta medicinal e alimento para a fauna.

Planta medicinal e alimento para a fauna (1)

6 Melífera. (1) Alimento para a fauna (3)

$36 \quad 16$ Produção de madeira, melífera, alimento fauna. (3)

4 Produção de madeira e alimento para a fauna

6 Produção de madeira e alimento para a fauna (2)

2 Planta medicinal e alimento para a fauna (2)

11 Alimento para a fauna. (1) Planta medicina. (3)

2 Produção de madeira e alimento para a fauna

$4 \quad$ Planta medicinal e alimento para a fauna

$4 \quad$ Produção de madeira (1)

Produção de madeira (1)

Produção de madeira e alimento para a fauna (1)

Planta medicinal e alimento para a fauna (4)

(1) Lorenzi (2002a); (2) Lorenzi (2002b); (3) IBGE (2002); (4) Silva Júnior (2005b); (5) SEMARH (2005); (6) Costa (2008); (7) Nogueira et al. (2004). 


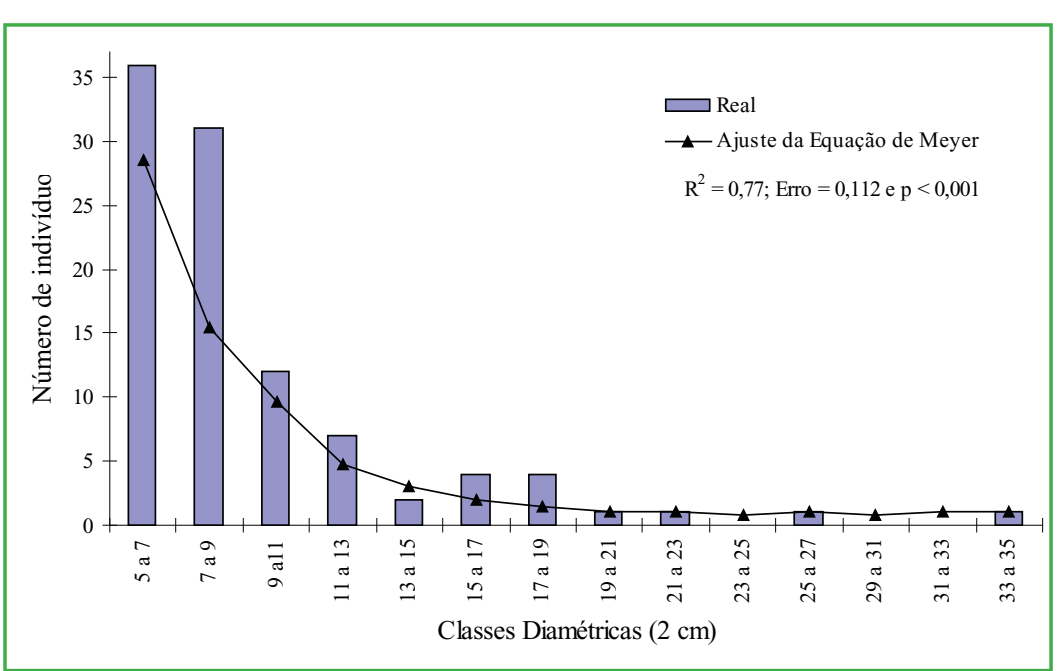

FIGURA 1: Distribuição diamétrica do cerrado sensu stricto em área da APA de Cafuringa, DF.

Uma curva de decaimento típica também pode ser observada na comunidade vegetal da floresta de galeria (Figura 2). As curvas mostram significância dos parâmetros estatísticos determinados, encontrados pela equação de Meyer (cerrado sensu stricto: $\mathrm{R}^{2}>0,77$, erro $<0,112$ e $P<0,001$; floresta de galeria: $\mathrm{R}^{2}>0,73$, erro $<0,179$ e $P<0,001)$, nos dois ajustes tanto para cerrado sensu stricto $\left(\mathrm{y}=15,587 \cdot \mathrm{e}^{-0,2551 \cdot \mathrm{x}}\right)$ como para a floresta de galeria $\left(y=33,646 \cdot \mathrm{e}^{-0,230 \cdot x}\right)$.

Os coeficientes de determinação $\left(\mathrm{R}^{2}\right)$ só não foram maior pela maior frequência de indivíduos entre as classes diamétricas de 15 a $19 \mathrm{~cm}$ para o cerrado sensu stricto, e 17 a $29 \mathrm{~cm}$ para a floresta de galeria, confirmando uma provável existência de uma intervenção antrópica ou natural nas classes anteriores aos intervalos citados, corroborando observações realizadas por Franceschinelli e Bawa (2005) e Soares et al. (2006).

Em forma semelhante ao cerrado sensu stricto, a análise dendrométrica das árvores da floresta de galeria resultou em área basal de $2,67 \mathrm{~m}^{2}$ para 155 indivíduos arbóreos (Tabela 1). Por estimativa esse valor para hectare corresponderia a $5,34 \mathrm{~m}^{2}$. ha $^{-1}$. Estes valores são baixos quando comparados com outras áreas de florestas de galeria localizadas relativamente próximas à área de estudo. Para as florestas de galeria do córrego dos Macacos foi registrado um valor de 26,44m ${ }^{2}$-ha ${ }^{-1}$ (Imaña-Encinas et al., 1995), 21,0m².ha-1 do córrego Capãozinho (Paula et al., 1993), 23,93 $\mathrm{m}^{2} \cdot \mathrm{ha}^{-1}$ para o rio São
Bartolomeu (Paula et al., 1990), 38,2 $\mathrm{m}^{2} \cdot \mathrm{ha}^{-1}$ do rio Pitoco (Silva Júnior, 2005b). O baixo valor em termos de área basal se explica em função da região ter sofrido forte intervenção antrópica no passado.

As espécies Ocotea corymbosa e Protium heptaphyllum apresentaram as maiores densidades populacionais, com 17 e 11 indivíduos arbóreos respectivamente. Em relação ao DAP médio, 13 espécies (26\%) mostraram possuir valores superiores a $15 \mathrm{~cm}$, e 27 espécies (54\%) valores inferiores a $10 \mathrm{~cm}$. Na Figura 2 mostra-se a distribuição diamétrica dos DAPs das 155 árvores da floresta de galeria, em intervalos de classe de $2 \mathrm{~cm}$.

As 155 árvores com DAP a partir de $5 \mathrm{~cm}$, na floresta de galeria, extrapoladas para hectare corresponderiam a 310 árvores. Na floresta de galeria do Rio São Bartolomeu (Distrito Federal), foram inventariadas 649 árvores com DAP a partir de $5 \mathrm{~cm}$ (Paula et al., 1990) e no córrego Capãozinho (Sobradinho, Distrito Federal) constataram-se 568 árvores com DAP a partir de $5 \mathrm{~cm}$ (Paula et al., 1993). Em um hectare da floresta de galeria do córrego dos Macacos, município de Santo Antônio do Descoberto (Goiás) próximo ao Distrito Federal, foram registradas 1.741 árvores (Imaña-Encinas et al., 1995; Paula et al., 1996). Consequentemente, pode-se deduzir que a densidade arbórea do fragmento da floresta de galeria do ribeirão Dois Irmãos estudado, é bastante baixa, comparada aos padrões descritos na literatura. 


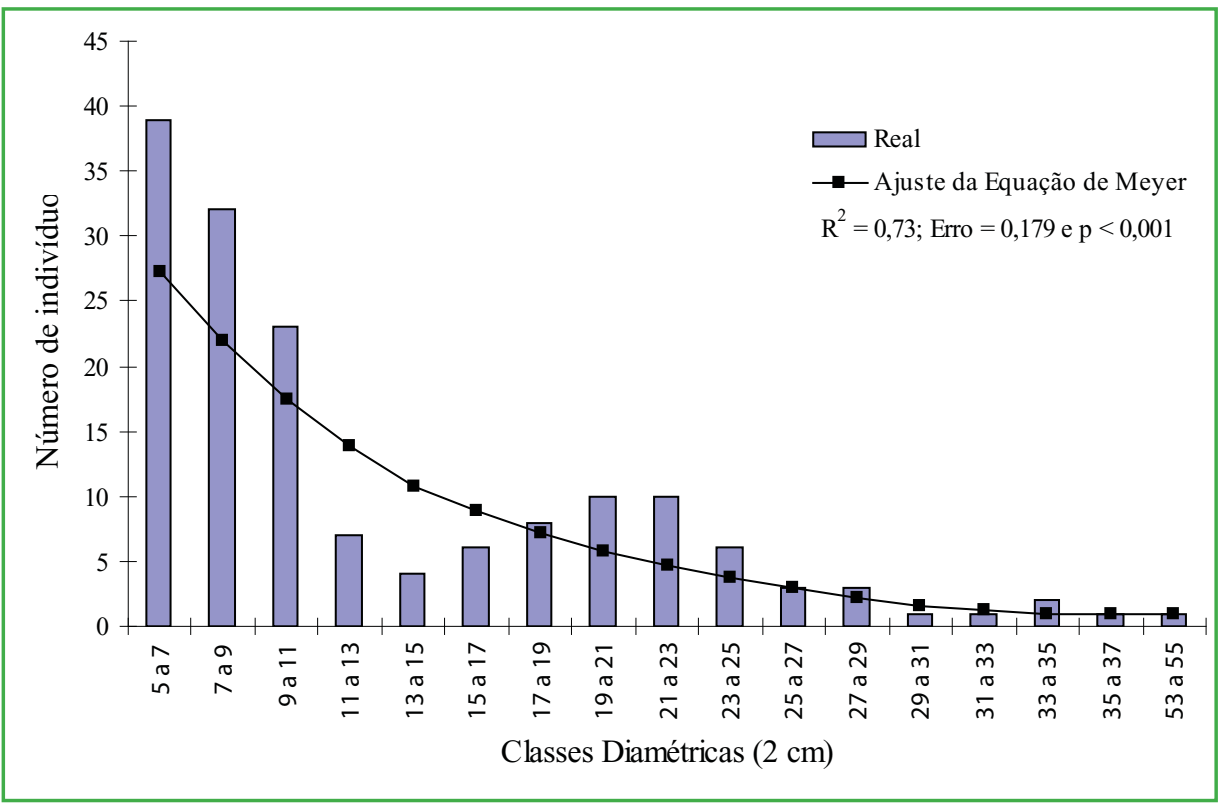

FIGURA 2: Distribuição diamétrica da floresta de galeria em área da APA de Cafuringa, DF.

Analisando, interpretando e comparando os resultados obtidos no presente estudo, com os constantes na literatura consultada e citada no texto, conclui-se que do ponto de vista florístico e da distribuição diamétrica, a vegetação da área do cerrado sensu stricto, sofreu forte interferência antrópica nas diversas classes diamétricas. Esse fato também se constatou na floresta de galeria do ribeirão Dois Irmãos.

\section{Referências}

Assunção, A. G.; Felfili, J. M. 2004. Fitossociologia de um fragmento de cerrado sensu stricto na APA do Paranoá, DF, Brasil. Acta Botanica Brasilica, 18 (4): 903-909.

Costa, J. F. O. 2008. Immunomodulatory activity of extracts from Cordia superba Cham. and Cordia rufescens A. DC. (Boraginaceae), plant species native from Brazilian Semi-arid. Revista Brasileira de Farmacognosia, 18 (1): 11-15.

Felfili, J. M. 1997. Diameter and height distribution in a gallery forest tree community and some main species in central Brazil over a six-year period (1985-1991). Revista Brasileira de Botânica, 20 (2): 155-162.

Felfili, J. M.; Silva Júnior, M. C.; Rezende, A. V.; Machado, J. W. B.; Walter, B. M. T.; Silva, P. E. N. da; Hay, J. D. 1993. Análise comparativa da florística e fitossociologia da vegetação arbórea do cerrado sensu stricto na Chapada Pratinha, DF-Brasil. Acta Botanica Brasilica, 6 (2): 27-46.

Felfili, J. M.; Silva Júnior, M. C.; Sevilha, A. C.; Fagg, C. W.; Walter, B. M. T.; Nogueira, P. E.; Rezende, A. V. 2004. Diversity, floristic and structural patterns of cerrado vegetation in Central Brazil. Plant Ecology, 174: 37-46.
Franceschinelli, E. V.; Bawa, K. S. 2005. The post-fire effects on the outcrossing rate of a Brazilian savannah shrub, Helicteres sacarolha A.St.-Hil. Revista Brasileira de Botânica, 28 (1): 163-170.

IBGE. 2002. Árvores do Brasil Central: Espécies da região geoeconômica de Brasília. Diretoria de Geociências, Rio de Janeiro, Brasil, 417pp.

Imaña-Encinas, J.; Paula, J. E. de. 2003. Análise da vegetação do cerrado do município de Santa Quitéria - Maranhão. Brasil Florestal, 22 (78): 33-41.

Imaña-Encinas, J.; Paula, J. E. de; Kleinn, C. 1995. Wood biomass of one gallery forest. Pesquisa Agropecuária Brasileira, 30 (4): 421-427.

Lorenzi, H. 2002a, Árvores brasileiras: Manual de identificação e cultivo de plantas arbóreas do Brasil. v.1. Instituto Plantarum, Nova Odessa, Brasil, 368pp.

Lorenzi, H. 2002b, Árvores brasileiras: Manual de identificação e cultivo de plantas arbóreas do Brasil. v.2. Instituto Plantarum, Nova Odessa, Brasil, 368pp.

Nogueira, R. C.; Paiva, R.; Castro, A. H. de; Vieira, c. V.; Abbade, L. C.; Alvarenga, A. A. 2004. Germinação in vitro de murici-pequeno (Byrsonima intermediaria A. Juss.). Ciência Agrotecnologia, 28 (5): 1053-1059.

Meyer, H. A. 1952. Structure, growth and drain in balanced unevenaged forests. Journal of Forest, 50: 85-92.

Paula, J. E. de; Imaña-Encinas, J.; Mendonça, R. C.; Leão, D. T. 1990. Estudo dendrométrico e ecológico de mata ripária da região Centro-Oeste. Pesquisa Agropecuária Brasileira, 25 (1): 43-55.

Paula, J. E. de; Imaña-Encinas, J.; Pereira, B. A. S. 1993. Inventário de um hectare de mata ripária do córrego Sobradinho-DF. Pesquisa Agropecuária Brasileira, 28 (2): 143-152.

Paula, J. E. de; Imaña-Encinas, J.; Pereira, B. A. S. 1996. Parâmetros volumétricos e da biomassa de mata ripária do córrego dos Macacos. Cerne, 2 (2): 91-105. 
Paula, J. E. de; Imaña-Encinas, J.; Sugimoto, N. 1998. Levantamento quantitativo de três hectares de vegetação de cerrado. Pesquisa Agropecuária Brasileira, 33 (5): 613-620.

Rezende, A. V.; Vale, A. T.; Sanquetta, C. R.; Figueiredo Filho, A.; Felfili, J. M. 2006. Comparação de modelos matemáticos para estimativa do volume, biomassa e estoque de carbono da vegetação lenhosa de um cerrado sensu stricto em Brasília, DF. Scientia Forestalis, 17: 65-76.

Santos, T. C. C.; Câmara, J. B. D. 2002. GEO Brasil 2002, perspectivas do meio ambiente no Brasil. IBAMA, Brasília, Brasil, 447pp.

SEMARH - Secretaria de Meio Ambiente e Recursos Hídricos. 2005. APA de Cafuringa, a última fronteira natural do DF. Governo do Distrito Federal, SEMARH, Brasília, Brasil, 544pp.
Silva Júnior, M. C. da. 2005a. 100 Árvores do cerrado: Guia de campo. Rede de Sementes do Cerrado, Brasília, Brasil, 278pp.

Silva Júnior, M. C. da. 2005b. Fitossociologia e estrutura diamétrica na mata de galeria do Pitoco na Reserva Ecológica do IBGE, DF. Cerne, 11 (2): 147-158.

Soares, J. J; Souza, M. H. A.; Lima, M. I. S. 2006. Twenty years of post-fire plant succession in a "cerrado", São Carlos, SP, Brazil. Brazilian Journal of Biology, 66 (2b): 587-602.

StatSoft, 1997. Statistic for Windows 5.1. Tulsa, USA: StatSoft Inc., CD-Rom

TROPICOS, 2008. Disponível em <http://www.tropicos.org/>. Acessos em 05 março de 2008 e 21 dezembro de 2008.

UNESCO. 2000. Vegetação no Distrito Federal - Tempo e espaço. UNESCO, Brasília, Brasil, 74pp. 